\title{
1 UAV Bridge Inspection through Evaluated 3D Reconstructions
}

2 Siyuan Chen ${ }^{\mathrm{a}}$, Debra F. Laefer ${ }^{\mathrm{b}}$, Eleni Mangina ${ }^{\mathrm{c}}$, Iman Zolanvari ${ }^{\mathrm{d}}$, Jonathan Byrne ${ }^{\mathrm{e}}$

$3 \quad{ }^{\text {a }}$ Univ. College Dublin, School of Civil Engineering, Urban Modelling Group, Belfield, Dublin 4, IE

$4 \quad$ b New York Univ., Center for Urban Science + Progress, 370 Jay St., 12th Fl, Brooklyn, NY 11201

$5 \quad{ }^{\mathrm{c}}$ Univ. College Dublin, School of Computer Science, Belfield, Dublin 4, Ireland

6 d Univ. College Dublin, School of Civil Engineering, Urban Modelling Group, Belfield, Dublin 4, IE

$7 \quad{ }^{\mathrm{e}}$ Univ. College Dublin, School of Civil Engineering, Urban Modelling Group, Belfield, Dublin 4, IE

$8 \quad *$ Corresponding Author: debra.laefer@nyu.edu

10 Abstract

11 Imagery-based, three-dimensional (3D) reconstruction from Unmanned Aerial Vehicles (UAVs)

12 holds the potential to provide safer, more economical, and less disruptive bridge inspection. In

13 support of those efforts, this paper proposes a process using an imagery-based point cloud. First,

14 a bridge inspection procedure is introduced, including data acquisition, 3D reconstruction, data

15 quality evaluation, and subsequent damage detection. Next, evaluation mechanisms are proposed

16 including checking data coverage, analysing points distribution, assessing outlier noise, and

17 measuring geometric accuracy. In this final aspect, the "Guide to the Expression of Uncertainty

18 in Measurement" was used. The overall approach is illustrated in the form of a case study with a

19 low-cost UAV. Areas of particular coverage difficulty involved slim features such as railings,

20 where obtaining sufficient features for image matching proved challenging. Shadowing and large

21 tilt angles hid or weakened texturing surfaces, which also interfered with the matching process. 


\section{Introduction}

26 Bridges are important infrastructure components that must be properly maintained to ensure public

27 safety and for which regular inspection is a critical component. Inspection approaches are to some 28 extent dictated by local practice. For example, Ireland's I-STR-6510 requires "ground level 29 inspections" be conducted every two years and a "thorough inspection" once every six years 30 (RAIU 2010). In the United Kingdom (UK), a "general inspection" should be undertaken every 31 one to three years according to the standard "Examination of Bridges and Culverts

32 NR/SP/CIV/017" (Sterritt 2009). Similarly, in the United States (US), a bridge should be inspected 33 every two years according to the American Association of State Highway and Transportation 34 Officials (AASHTO) requirement (AASHTO 1970). Traditionally when inspecting bridges, there 35 is a choice between using an Aerial Work Platform (AWP), an under-bridge inspection vehicle, 36 ladders, or ropes for access. Irrespective of the method used, the associated costs and dangers 37 remain challenges. AWPs and inspection vehicles are likely to require road lane closures, and the 38 equipment used is expensive to maintain and run, while ropes require a high level of training and 39 expertise to be used safely. To date, there has yet to be a rapid and cost-effective method that does 40 not require bridge closure and is able to generate a permanent record. To address that deficit, this 41 paper considers the feasibility and limitations of using an unmanned aerial vehicle (UAV) for

42 documentation from which subsequent inspection can be conducted through a three-dimensional 43 (3D) reconstruction. The paper presents recent efforts in this area followed by a new evaluation 44 framework for 3D reconstruction. The usefulness and importance of this evaluation framework is 45 shown in a case study that demonstrates the proposed workflow for data acquisition, model 46 reconstruction, and data quality determination. 


\section{Inspection Approaches}

49 Currently, visual inspection is the primary form of bridge inspection. This may involve in-person

50 inspection, fixed sensors, or camera-based monitoring. Since each has its limitations, significant

51 interest has emerged in using UAVs, as a means to provide faster, cheaper, safer, and more flexible

52 data acquisition, along with generation of an objective digital record, instead of in-person visual

53 assessment, as reported in recent state-of-the-art reviews by Chen et al. (2016) and Hassanalian

54 and Abdelke (2017). The following concentrates on recent efforts to use remote sensing for 55 inspection.

\section{Remote Sensors and Camera-based Inspection}

58 Remote sensors and camera-based inspection can provide continuous bridge evaluation data

59 through permanent deployment, thereby minimizing the safety problems of in-person inspections

60 and the impacts of affiliated bridge closures. To this end, Jahanshahi et al. (2011) introduced an

61 image-based system for bridge inspection (Figure 1a) where on-site imagery was transmitted via

62 cable to an off-site database, and a computer-vision based process was used to reduce

63 inconsistencies in individual inspections. At a working distance of $3 \mathrm{~m}$, with a Canon PowerShot

64 A610 digital camera, the reported minimum measurable feature was $0.57 \mathrm{~mm}$. However, the high

65 costs and relatively fixed inspection ranges affiliated with stationary cameras continue to curtail

66 the popularity of this approach. According to a report published by the Minnesota Department of

67 Transportation (Lueker and Marr 2014), the cost for setting up a continuous bridge monitoring

68 system is around $\$ 25,000$ for the first year with $\$ 1,000$ per year for annual maintenance. 

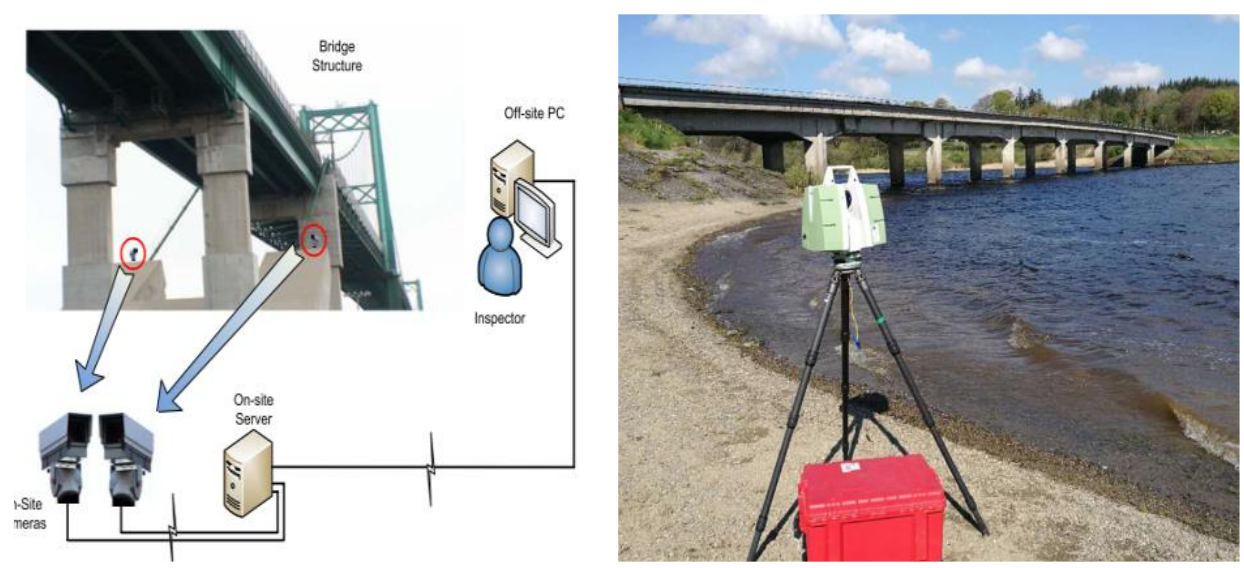

Fig. 1. Remote sensors and cameras inspection. (a) Camera inspection

(Jahanshahi et al. 2011); (b) Terrestrial laser scanner (TLS)

73 To use such a monitoring system in a more efficient way, mobile devices have been developed and

74 deployed. Examples include the work by Nishimura et al. (2012) where a hybrid camera system

75 was fixed atop a moving vehicle This system combined a fixed angle camera for detailed detection

76 and a 360-degree camera for panoramic data recording. However, this system can only be applied

77 in vehicle-accessible areas and is, thus, not fit for underbridge inspection or for documenting

78 distant features such as cables and towers. Terrestrial laser scanning (TSL) is another commonly

79 used approach that can provide high-quality 3D data for bridge damage detection, such as surface

80 loss or cracks (Truong-Hong and Laefer 2015; Truong-Hong et al. 2016). However, those scanners

81 are relatively expensive (typically starting at $\$ 25,000$ ) and need a flat base and clear line of site

82 (Figure 1b), which may not be available. Moreover, as the scanner's location is fixed during

83 scanning, the line of sight nature of the technology may potentially result in occlusions where

84 objects are located between the scanner and the target object or when the scene geometry causes

85 self-shadowing (see Figure 2) [Hinks et al. 2009]. 

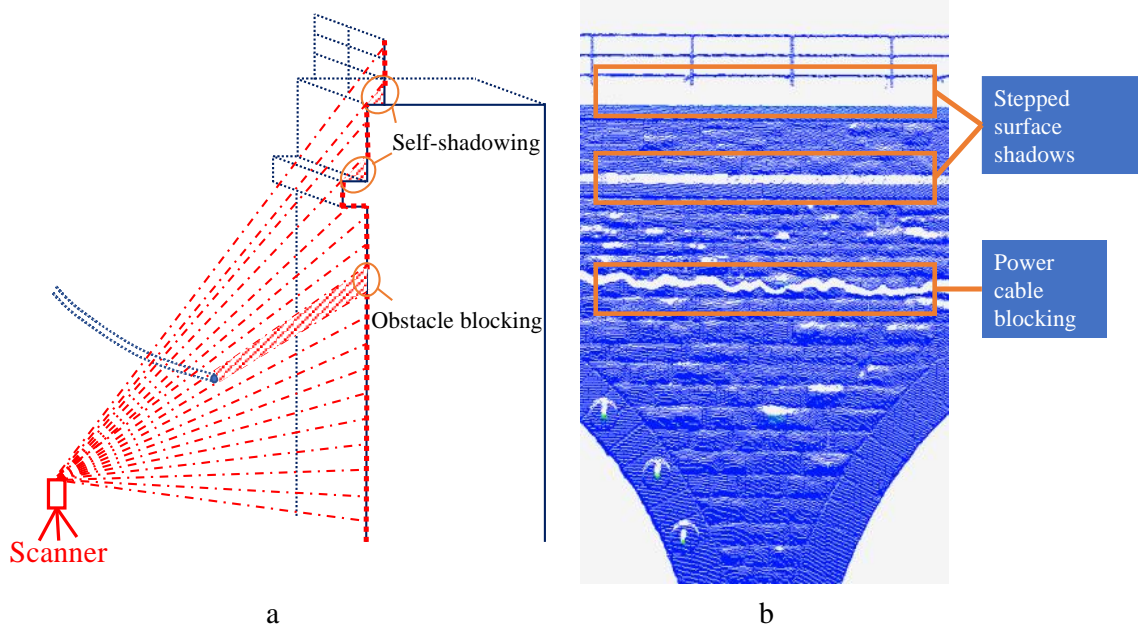

88 Fig. 2. Missing data phenomenon in TLS scans. (a) Schematic of occlusion and self-shadowing problem; (b) Point cloud from TLS data

\section{Unmanned Aerial Vehicle (UAV) Inspection}

92 As a possible alternative, UAV-based inspections can offer the combined advantages of robot

93 inspection and remote sensor inspection. As such, the topic has received significant interest for

94 baseline documentation and surface evaluation of bridges (Yin et al. 2015), roads (Díaz-Vilariño

95 et al. 2016) and buildings (Fernandez Galarreta et al. 2014). Compared to traditional inspection

96 methods, UAV-based inspection has clear advantages. Firstly, in hard to reach areas, such as cable

97 towers and deck bottoms, UAV-based access is less restricted by distance and angle. So better site

98 visibility and optimized views can be acquired (Kim et al. 2015), especially where computer-based

99 path planning is employed to maximize data capture coverage (Bircher et al. 2015). Secondly,

100 UAVs present a significant financial advantage. For example in 2015, Chan et al. (2015)

101 introduced a UAV system for bridge inspection that employed an aerial light detection and ranging

102 (LiDAR) sensor that cost about $\$ 6,000$, which was less than a quarter of the cost of in-person

103 methods. More recently, Byrne et al. (2017a) presented a solution to employ UAV-based aerial 
104 video footage for building surveying, with equipment costing less than $\$ 1,000$. Thirdly, UAVs can

105 carry a wide range of task-specific sensors, including RGB cameras, laser scanners, thermal

106 cameras, hyperspectral cameras, and aperture radars, for different inspection purposes (Chen et al.

107 2016).

108

109 Until relatively recently, laser scanners were able to provide high quality 3D point clouds only

110 with relatively expensive and heavy equipment needing to be mounted on fixed-wing UAVs

111 (Wallace et al. 2012). This was problematic, as effective bridge inspection requires outstanding

112 hovering capabilities and manoeuvrability around piers and even between trusses, which

113 necessitates a small, multi-rotor UAV. Due to weight and expense, imagery has been favoured for

114 UAV-based bridge inspection but not without difficulties. Kim et al. (2015) presented such a

115 camera-based, UAV system for concrete bridge surface crack detection. In their research, a

116 morphological algorithm was designed for detecting and measuring crack widths but resulted in a

117 highly variable error (3\%-50\%). However, in this fast-changing field, significant improvements

118 occur frequently in terms of both hardware and software. As an example, Escobar-Wolf et al.

119 (2017) employed a thermal camera for under-surface delamination and hole detection. In their case

120 study, they generated thermal and visible images for a $968 \mathrm{~m}^{2}$ area, from which $14 \mathrm{~m}^{2}$ of

121 delamination was identified - an overall accuracy of about $95 \%$ compared to direct contact

122 hammer sounding data. Based on the current technology and the applications of UAVs in bridge

123 inspection, there are two aspects of aerial data collection that can improve results, which are

124 considered as part of the proposed methodology:

125 1. Separation of the requirements and the necessary processes: Bridge inspection is 126 requirement driven, with the desired information scope and type typically dictated by the 

specific bridge. As such, every aerial data collection mission should start with the identification of the requirements to which any generic or proprietary process must be applied. The specified process forms the foundation of how to (1) achieve the desired data collection, (2) add value over traditional methods, and (3) maintain high safety standards during the execution.

2. Assessment of the flight process: Each operation is unique and comes with specific operational variables that must be considered to achieve a safe and legally compliant flight mission.

\section{Methodology}

137 To achieve a systematic and reliable bridge inspection, a UAV-based inspection framework is 138 needed, as proposed in Figure 3. As will be explained in the following subsections, this involves 139 four main tasks: (1) data acquisition; (2) 3D reconstruction; (3) quality evaluation of the 3D 140 reconstruction; and (4) damage detection.
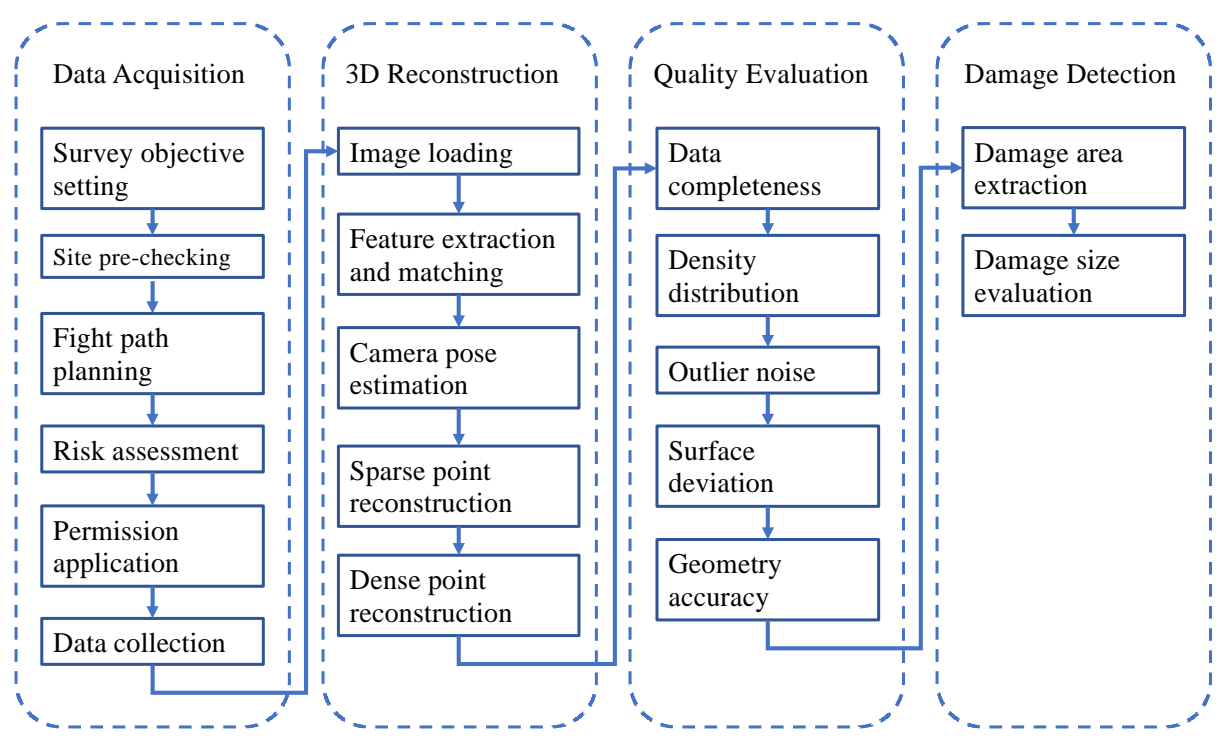


\section{Data Acquisition}

145 The task of data acquisition includes site pre-checking, fight plan drafting, risk assessment,

146 permission application, and on-site data collection. Each step has its own requirements as

147 introduced in the Table 1.

149 Table 1. Procedures for UAV inspection

\begin{tabular}{ll}
\hline Workflow & Steps \\
\hline Survey objective setting & $\begin{array}{l}\text { Determine which areas are to be covered and what } \\
\text { information needs to be collected. } \\
\text { Site pre-checking }\end{array}$ \\
$\begin{array}{l}\text { Become familiar with the basic geographical information } \\
\text { of the target and its surroundings to know the traffic } \\
\text { frequency of nearby roads and distances to those roads, } \\
\text { surrounding buildings, and/or trees. }\end{array}$ \\
$\begin{array}{l}\text { Choose the flight path - including the take-off locations, } \\
\text { flight speeds and heights, distance to the object, camera } \\
\text { settings, and emergency landing places. Check the }\end{array}$ \\
$\begin{array}{l}\text { weather to avoid windy and raining days, and avoid peak } \\
\text { traffic hours. }\end{array}$ \\
$\begin{array}{l}\text { Reduce the risk of the accident by keeping a notable } \\
\text { distance from the survey target, vehicular traffic, people, }\end{array}$ \\
water, trees, power cables, and signal towers. \\
Obtain permissions from the landowner or site manager \\
and the aviation authority for the specified flight plan. \\
Permission application \\
Notify any potentially impacted populations about when \\
the aerial survey will start. Follow the devised flight plan \\
for data collection, if any emergency occurs, land the \\
UAV safely.
\end{tabular}

151 Among the Table 1 steps, the flight path planning arguably has the strongest impact on the data

152 quality, as it relates to light conditions, camera angle, offset distances, flight pattern, and degree

153 of overlap between images (Chen et al. 2017). While overlapping rates are rarely reported and

154 appear to be empirically selected, Paine and Kiser (2003) recommended $60 \% \pm 5 \%$ for endlap and

$15530 \% \pm 15 \%$ for sidelap. 
156 To better explain the relationship between camera angle and distance, the terminology Ground

157 Sampling Distance (GSD) is referred to in remote sensing as spatial resolution, which is used here

158 to describe the image quality. The GSD equals the distance between the centre of two consecutive

159 pixels on the target surface. Figure 4 shows the projection relationship of a simplified digital

160 camera system. In an orthographic projection, the GSD will be the same in the field. In a tilt

161 projection, the far end will have the maximum GSD value. This means that each pixel covers a

162 larger area in the corner D than in corner A, and the edge DC will have the maximal GSD of the

163 entire field of view (FOV). Figure 5 shows the relationship between the GSD value, the sensor

164 size [horizontal sensor size (HSZ) times vertical sensor size (VSS)], the focal length (f), the

165 working distance (WD) from the camera to the object, the camera tilt angle ( $\alpha \mathrm{t})$ from the camera

166 axis to the surface normal, and the resolution of the sensor [horizontal pixel numbers (HN) time

167 vertical pixel numbers $(\mathrm{VN})]$.

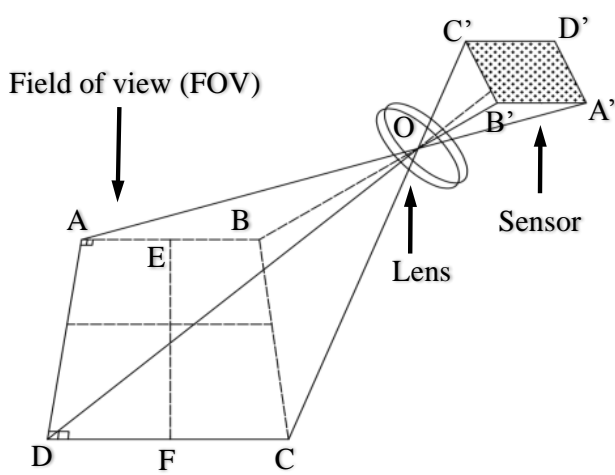

Fig. 4. Projection relationship 3D

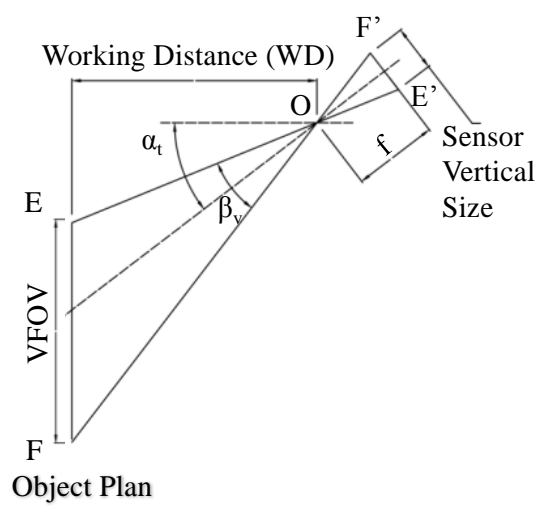

Fig. 5. Projection relationship two-dimensional (2D)

169 Based on the geometric relationship, the average GSD on the edge CD can be calculated by 170 equation (1). The vertical camera view angle $\beta v$ is defined in equation (2). 


$$
\mathrm{GSD}_{\text {max }}=\frac{W D \times \mathrm{HSS} \times \cos \frac{\beta_{v}}{2}}{f \times \mathrm{HN} \times \cos \left(\alpha+\frac{\beta_{v}}{2}\right)}
$$

$$
\beta_{v}=2 \times \tan ^{-1} \frac{V S S}{2 \mathrm{f}}
$$

174 Ideally, a smaller GSD value is better, but the FOV value should be considered as well, as a larger 175 FOV minimizes the number images required for data collection. The FOV value can be calculated 176 by equation (3) [see Byrne et al. 2017b for a further discussion of this point].

$$
\mathrm{FOV}=\frac{W D^{2} \times H S S}{2 f} \times\left(\frac{\cos \frac{\beta}{2}}{\cos \left(\alpha-\frac{\beta}{2}\right)}+\frac{\cos \frac{\beta}{2}}{\cos \left(\alpha+\frac{\beta}{2}\right)}\right) \times\left(\tan \left(\alpha+\frac{\beta}{2}\right)-\tan \left(\alpha-\frac{\beta}{2}\right)\right)
$$

180 For inspections, most data acquisition parameters are related to the device and are unalterable, 181 such as the sensor size, focal length, and pixel numbers. For example, with the DJI Phantom 4 $182 \mathrm{UAV}$, the sensor size is $6.17 \mathrm{~mm} \times 4.55 \mathrm{~mm}$, the focal length is fixed at $3.55 \mathrm{~mm}$, and the pixel 183 numbers are $4000 \times 3000$. Thus, the maximal GSD value and FOV value are only affected by the 184 working distance and the tilt angle. Figure 6 demonstrates calculating the FOV vs GSD chart for 185 DJI phantom 4, with respect to the tilt angle and offset distance. After calculation of the GSD and 186 FOV, an appropriate working distance and tilt angle can be selected to match the surveying 187 objective(s) for image collection. Once collected, imagery can be used for 3D model generation, as described in the next subsection. 


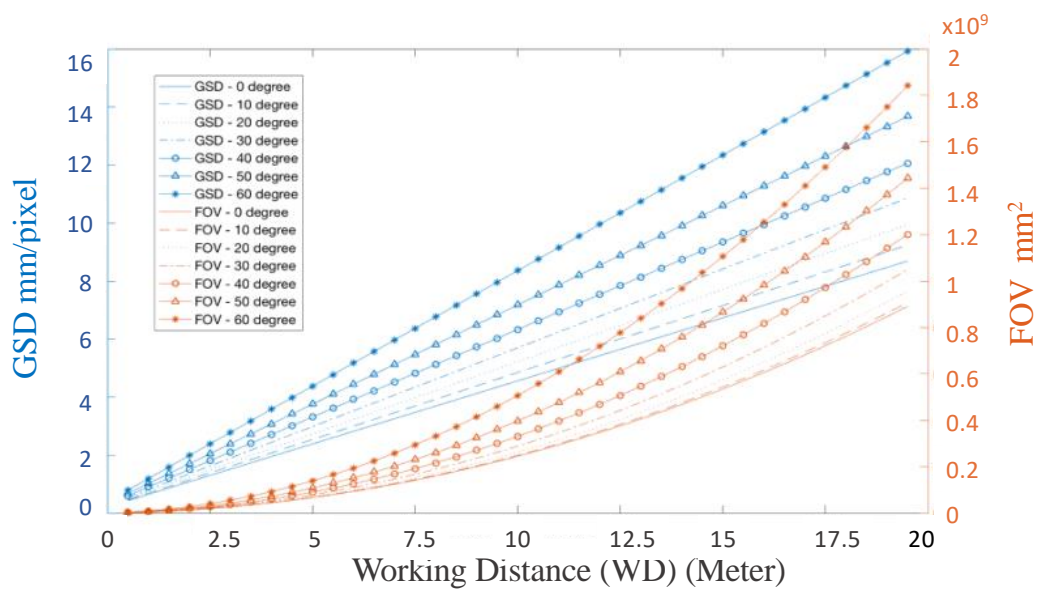

Fig. 6. FOV vs GSD

\section{3D Reconstruction}

193 Once imagery data are captured, they must be processed in a manner usable for the final

194 application. Traditionally, conventional camera inspections have concentrated on individual 2D

195 images, which precludes direct 3D location measurement and volumetric estimation (Eschmann et

196 al. 2013). Further manipulation to generate a 3D point cloud can be achieved through the Structure

197 from Motion (SfM) method, as first introduced by Ullman (1979). SfM utilizes images taken from

198 at least two viewpoints. By detecting key points in each image, the geometric relationship between

199 images can be calculated and used for triangulation, from which the depth information of key

200 points is derived and placed into a unique coordinate system. The approach can be decomposed

201 into (1) feature extraction and tracking, (2) pose estimation, (3) 3D point registration, and (4)

202 surface reconstruction (Szeliski 2010). A scale-invariant feature transform (SIFT) [Lowe 2004],

203 providing efficient feature extraction and bundle adjustment, was also applied to minimise the

204 cumulative drift errors (Schonberger and Frahm 2016). 
206 Those steps have been integrated in open source software like VisualSFM or OpenMVG and 207 commercial software like PhotoScan and Pix4D and used for forest mapping (Wallace et al. 2016), 208 geoscience surveying (Westoby et al. 2012), agriculture monitoring (Zarco-Tejada et al. 2014), 209 and urban modelling (Byrne and Laefer 2016). With respect to bridges, Hallermann et al. (2016)

210 presented a case study that illustrated the possibility of using UAVs for 3D bridge inspection.

211 However, published work in this area tends not to report evaluations of the quality of the full 212 reconstructed 3D point clouds, instead reporting evaluations only from further derived products 213 (e.g. crack identification).

214

\section{Data Quality Evaluation}

216 Generally, 3D reconstructed point clouds include defects such as missing data. This is caused by

217 line-of-site-based occlusions (Tagliasacchi et al. 2009), non-uniform data densities (Berger et al. 218 2014), inaccurate geometric positioning (Sargent, et al. 2007), surface deviations (Koutsoudis et 219 al. 2014), and outlier-based noise (Cheng and Lau 2017). Each defect type is illustrated in Figure 220 7. Despite the common occurrence of these types of problems, specific metrics to evaluate UAV221 generated 3D models have yet to be established. A review of 20 papers published between 2000 222 and 2017 related to UAV-based inspection with imagery based point clouds demonstrated that 223 only three of them considered any evaluation beyond subjective visual fidelity. Of those Byrne et 224 al. (2017b) proposed using inlier matching, as well as the final reconstruction, while Palmer et al. 225 (2015) and Koutsoudis et al. (2014) evaluated geometric distance errors. Notably despite the 226 rapidly growing popularity of UAV-based imagery 3D reconstructions, a broadly accepted set of 227 standards for evaluating the resulting 3D models has yet to established. To overcome this deficit, 228 the research herein will propose a rigorous evaluation method for assessing UAV-generated, 3D 
229 point clouds for the purpose of bridge inspection. For this, a series of functions has been designed

230 to consider each possible defect within the data evaluation flow chart (Figure 8), as explained in

231 the following sections. The results have been benchmarked against terrestrial laser scanner (TLS)

232 data, as that technology is widely used in surveying and considered to be accurate to the centimetre

233 level in building inspection (Quagliarini et al. 2017).

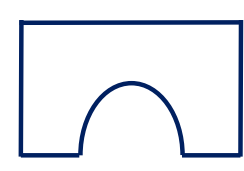

a
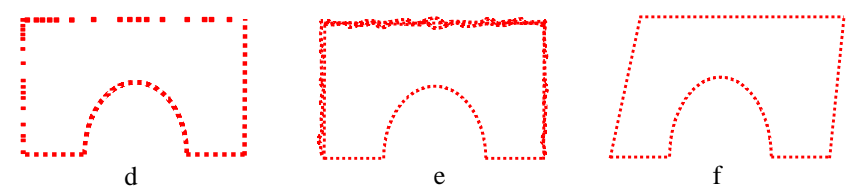

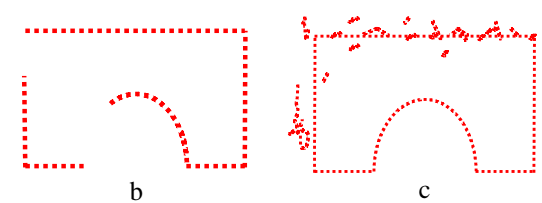

c

Fig. 7. Point cloud defects. (a) Real structure; (b) Incomplete data; (c) Outlier noise

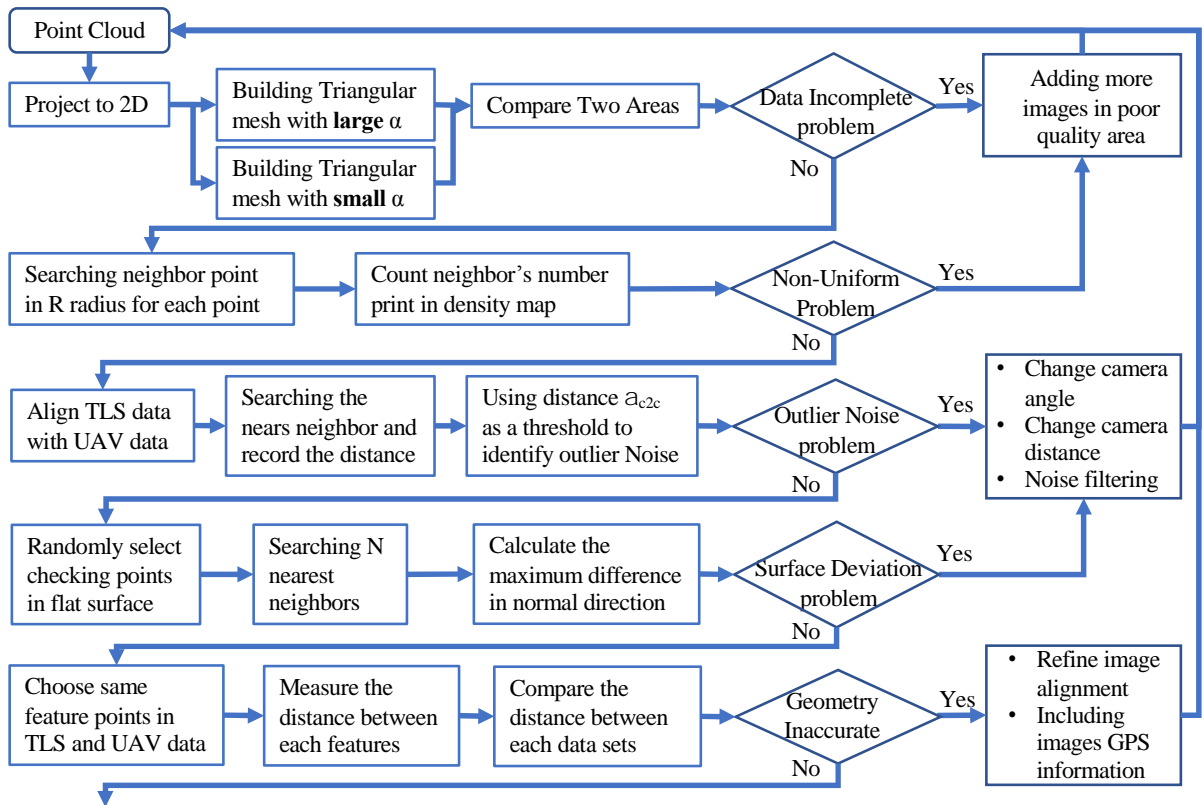

Further Analysis

Fig. 8. Flow chart of data evaluation 


\section{Evaluating Incomplete Data}

240 In terms of UAV-based reconstruction, the missing data problem persists in poorly overlapped

241 areas (Figure 9a), especially for slim or narrow portions of the structures (e.g. railings in Figure

$2429 b)$, since there are insufficient features for image matching. Increasing the extent of image overlap

243 can minimize this problem.

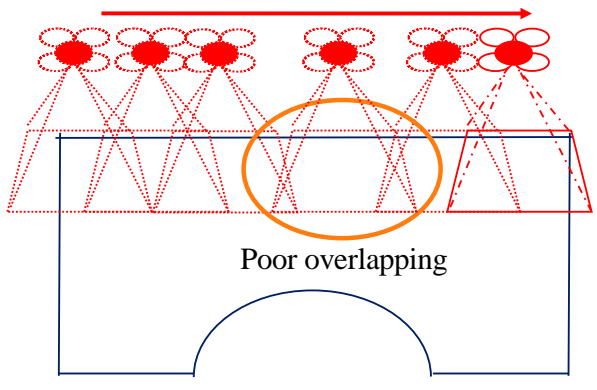

$\mathrm{a}$

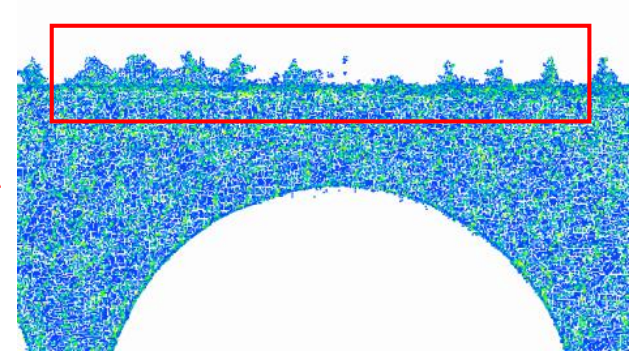

$\mathrm{b}$

245 Fig. 9. UAV-SFM data missing. (a) Poor overlapping; (b) Sample UAV data taken from 20m

247 For the purpose of quantifying the degree of data completeness, a 2D area evaluation method was

248 designed. This involves first projecting the testing surface data (Figure 10a) onto their normal

249 plane. Then, in the $2 \mathrm{D}$ projection plane, a triangular mesh is built between each point. The

250 threshold $\alpha$ is applied here to control the searching radius for mesh generation. For any point C,

251 within the radius $\alpha$, if any neighbour points exist, a triangular mesh will be generated for area

252 calculation. Thus, by controlling the threshold $\alpha \mathrm{p} 2 \mathrm{p}$, the area with and without incomplete

253 coverage can be calculated. To choose an appropriate $\alpha$, the average distance of any point to its

254 nearest neighbours must be measured. In this algorithm, 5\% of the points were randomly taken

255 from the original data as querying points and used in a nearest neighbour searching (NNS)

256 algorithm (Muja and Lowe, 2009) to find the closest point to each query point. Then, the average

257 Euclidean distance $\left(\beta_{\mathrm{ave}}\right)$ and standard deviation $\left(\beta_{\mathrm{std}}\right)$ of all pairs of query points and their closest 
258 neighbours are calculated. If $\alpha$ is much larger than $\beta_{\text {ave, }}$ then the incomplete area is included, as

259 shown in Figure 10b. Although not entirely accurate, because this mesh fills all the holes and fully 260 covers the structure, this meshed representation will be used as the ground truth for the purpose of 261 evaluation. If the $\alpha_{\mathrm{p} 2 \mathrm{p}}$ value is close to $\beta_{\text {ave }}$ and within $\pm \beta_{\text {std, }}$, then the mesh will ignore the 262 incomplete area and only represent the real data coverage, as shown in Figure 10c. By comparing 263 these two meshes, the degree of coverage can be measured to a reasonable level.

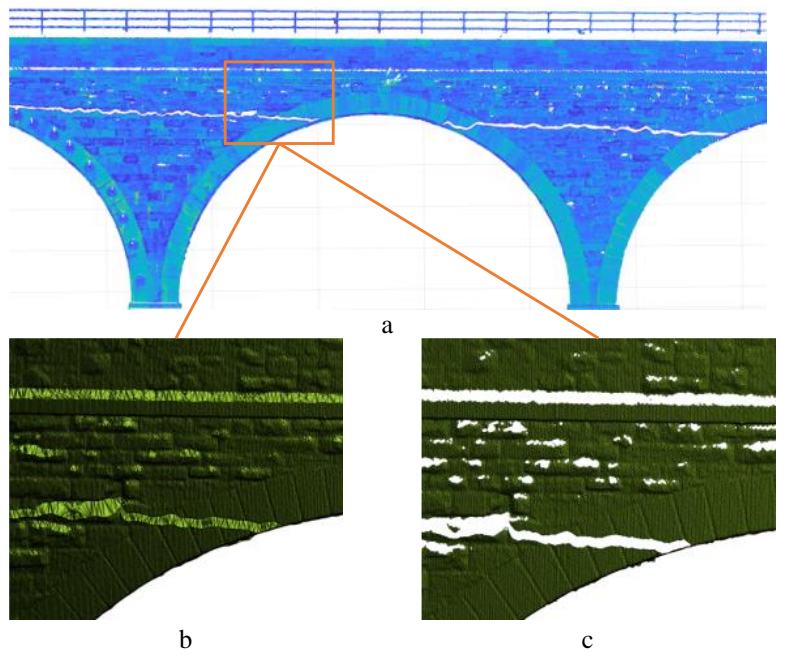

265 Fig. 10. Testing dataset $(\beta a v e=0.02 \mathrm{~m}, \beta \mathrm{std}=0.006 \mathrm{~m}) \mathrm{b}$. Mesh with incomplete area $(\alpha \mathrm{p} 2 \mathrm{p}=0.2)$ c. Mesh without incomplete area $(\alpha p 2 p=0.025 m)$

\section{Evaluating Non-uniform Distribution}

269 A non-uniformly distributed point cloud may have insufficient points in low-density areas, which

270 will cause problems for further analysis, such as point cloud simplification (Moenning and 271 Dodgson 2003) or surface reconstruction (Huang et al. 2009). The point distribution can be 272 measured easily by volume density. For each point, the number of neighbouring points in a 273 spherical neighbourhood of a defined radius $\mathrm{R}$ can be counted and presented in a density map. As 
274 illustrated in Figure 11, point A has 4 neighbour points in the searching area within a radius R, 275 while point $\mathrm{B}$ has 6 neighbours, and point $\mathrm{C}$ has 9.

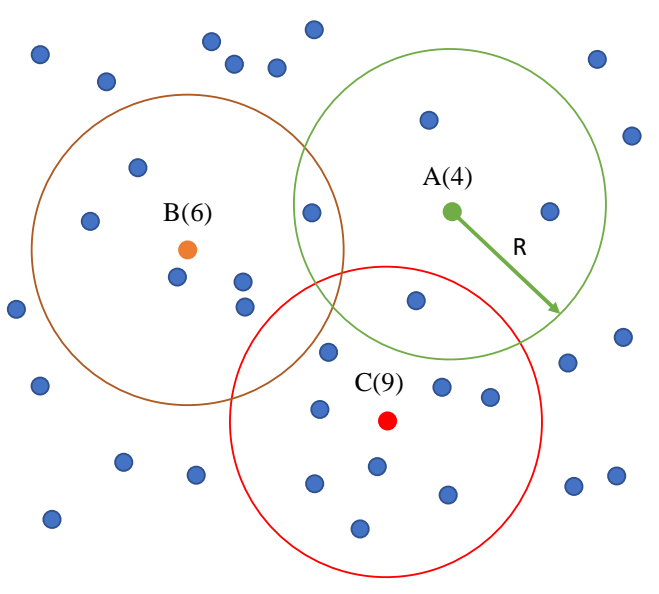

Fig. 11. Volume density

279 Evaluating Outlier Noise

280 Outlier noise usually appears around the boundary of the structure. One reason is that textureless

281 backgrounds (like sky) tend to confuse SfM approaches. For example, the railing area in Figure

28212 is poorly reconstructed, as the reconstruction algorithm treats the background (sky) as part of

283 the front object (bridge). For example, as the camera failed to fully observe the area beneath the

284 arch, many outliers appear around the border. Those outlier points will affect subsequent surface

285 reconstruction and generate floating artefacts around the object. Additionally, shadows and large

286 tilt angles weaken or hide the surface texture. 


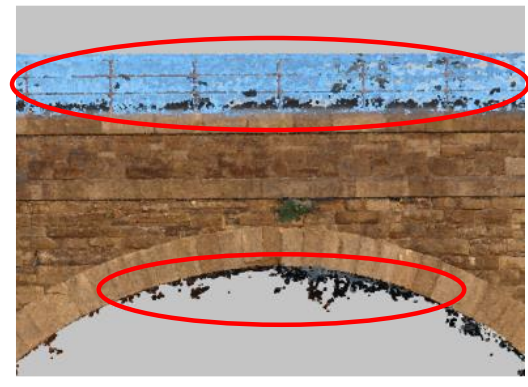

a

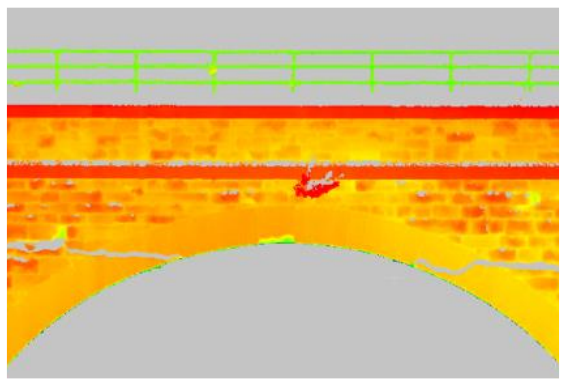

$\mathrm{b}$

Fig. 12. Outlier Points comparison. (a) UAV dataset; (b) TLS dataset

290 As the outlier problem is more significant in the imagery, the TLS dataset is considered as the 291 reference dataset and compared to the relative noise level in the UAV data. To do this, first the 292 UAV data are aligned to the TLS data using the Iterative Closest Point (ICP) algorithm (Besl and 293 McKay 1992). Then, the distance between specific points in each set is calculated. For each point 294 in the UAV dataset, a search is undertaken for its nearest neighbour point in the TLS dataset, and 295 the offset distance is recorded. An example of a cloud-to-cloud distance map is shown in Figure 296 13. By setting a threshold $\alpha_{c 2 c}$ to control the maximum distance, the outlier noise can be filtered 297 out, as shown in red in Figure 13. Here, $\alpha_{c 2 c}$ equals the mean distance $\lambda_{\text {ave }}$ plus two times the 298 standard deviation $\lambda_{\text {std. }}$ Using the total number of points to divide the outlier points number shows 299 the percentage of outlier noise.

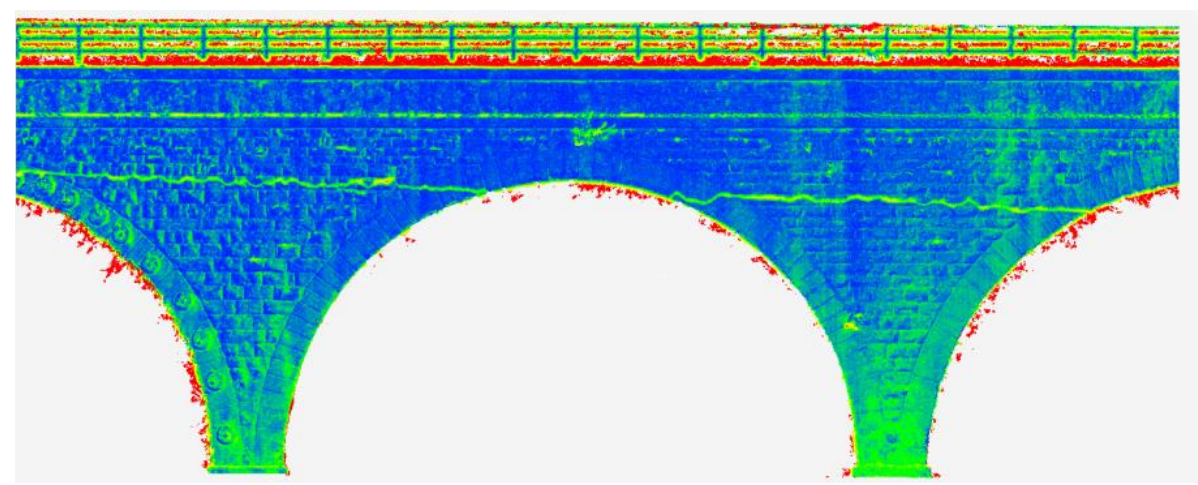

Fig. 13. Cloud to cloud distance map ( $\lambda$ ave $=0.04, \lambda \mathrm{std}=0.07, \alpha \mathrm{c} 2 \mathrm{c}=0.18)$ 
303 Theoretically, a surface should contain only one layer of points. Thus, the thickness of points along 304 a scanned surface should be close to zero, but the reality is otherwise. This is because the 305 reconstruction mechanisms are not completely accurate. Specifically, some points will deviate 306 from the real surface, which results in the point cloud surface presenting as if it is of a certain 307 thickness, despite its true planar nature. The thickness will cause problems for further mesh 308 generation, surface reconstruction, and retention of small details (Wolff et al. 2016). A method to 309 evaluate the point cloud surface deviation level involves selecting a few checkpoints to measure

310 the thickness and point distribution in the immediate neighbourhood. Choosing the checkpoint is 311 best done from a flat surface to avoid incorporating surface changes in the deviation. An example 312 is shown in Figure 14, where three checkpoints are selected within a defined neighbourhood of 1 $313 \mathrm{~cm}^{2}$ in the XY direction. The difference between Z-max and Z-min is the thickness at that location.

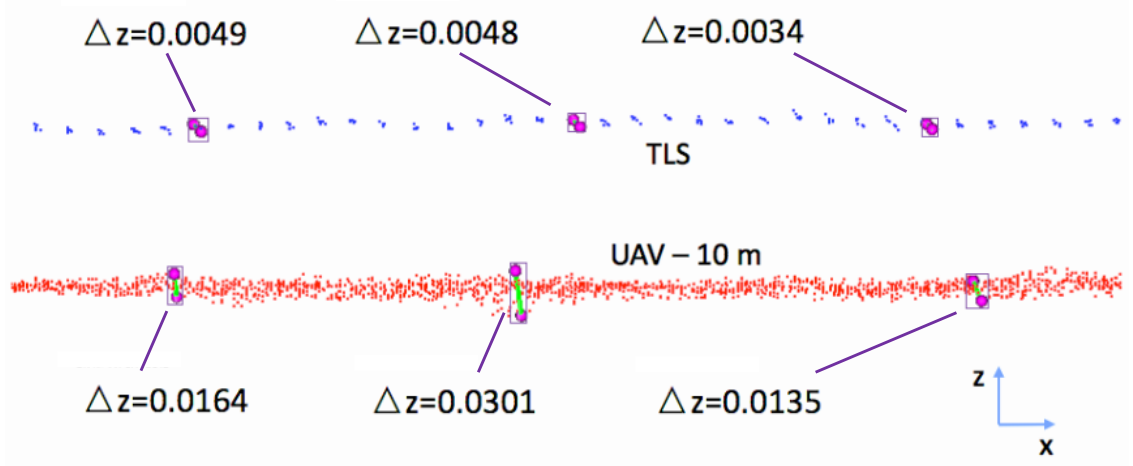

Fig. 14. Surface deviation

\section{Evaluating Geometric Accuracy}

318 Geometric accuracy is important for engineering inspection, especially for deformation monitoring 319 and quantifiable damage assessment. One method to do this involves measuring the point-to-point 320 distance of specified feature pairs (Koutsoudis et al. 2014). This requires choosing a few visually 
321 recognizable feature points (e.g. a corner or colour mark). By measuring the relative distance of

322 the same feature pairs in the different datasets, the relative accuracy between the different datasets

323 can be measured.

324

\section{Damage Evaluation}

326 Compared to image based 2D inspection, reconstructed 3D point clouds provide depth information 327 for holes and cracks making volumetric damage calculation possible, which is important for 328 structural health evaluation. To achieve that, the damaged area needs to be extracted from the

329 dataset. This can be completed by means of manual segmentation or using an auto-clustering 330 algorithm, such as K-means or DBSCAN. Within the extracted boundary, volume calculation can 331 be done by filling the space with random points and generating a triangular mesh from which the 332 volumetric calculation can be done.

\section{Case Study}

335 To demonstrate the proposed procedure, a field test was conducted of the Boyne Viaduct Bridge 336 (Figure 15), located in Drogheda, Ireland. The bridge was selected because of its location beyond 337 the restricted air space of Dublin Airport and its clear line of sight for TLS inspection. The bridge 338 is $30 \mathrm{~m}$ high, comprised of 15 masonry spans (12 on the south and 3 on the north side), as well as 3393 girder spans of wrought-iron. After site pre-checking, risk assessment, and permission 340 application (Table 1), arches No. 1 to No. 6 on the southern side were selected as the focus of the 341 survey. Flight permission was not possible for arches No. 7 to No. 12 due to potential UAV-risks 342 to pedestrian, vehicles, and the adjacent railway. Furthermore, the northern abutment was located 343 on private property for which requested access was denied. The survey was conducted at 5:30 a.m. 
344 of May of 2017 and lasted for 40 minutes. The TLS unit was located on the south bank where

345 permission was obtainable.

\section{Data Acquisition}

\section{UAV Data Collection}

349 A relatively low end UAV in the form of a DJI Phantom 4 quadrotor (Figure 16) was employed 350 with a 12-megapixel (4000x3000) digital camera. This commercial unit was augmented with a 3351 axis stabilization gimbal. While more expensive UAVs and cameras are available, the purpose of 352 this flight was to show the proposed framework in a real-life scenario.

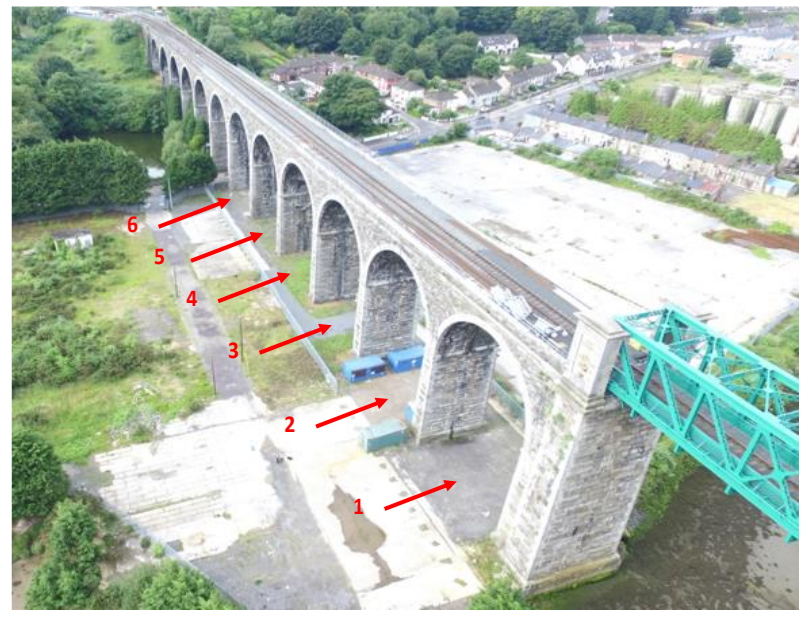

Fig. 15. Boyne Viaduct Bridge showing the south side of arches 1-12

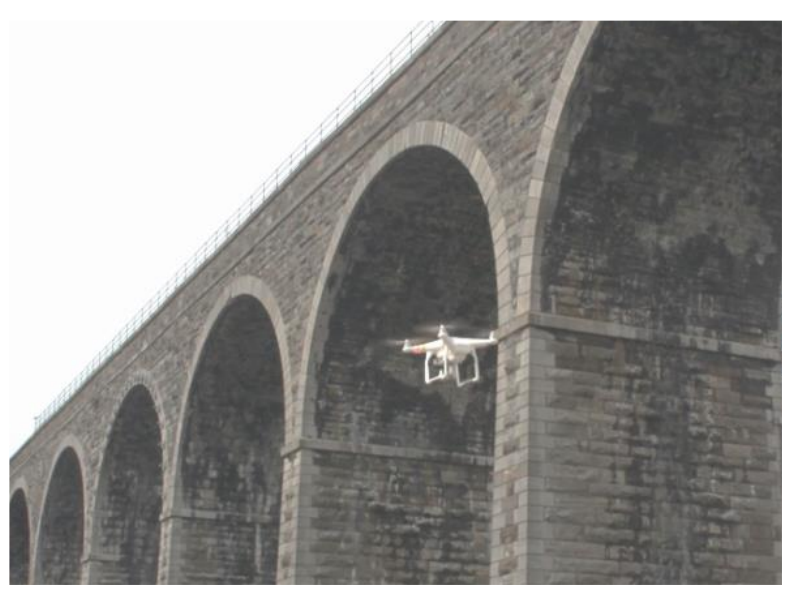

Fig. 16. UAV showing the south side of arches 1-6

354 The flight trajectory was pre-designed as per Figure 17. On each side of the bridge's southern end, 355 take offs $\mathrm{A}$ and B included 3 flight paths with angles ranging from $0^{\circ}$ to $45^{\circ}$ and offset distances 356 of $20 \mathrm{~m}$ to $40 \mathrm{~m}$ (Table 2). To obtain additional details for surface deterioration, a third take off 357 was undertaken. Arch No. 5 was selected as the target, because a small spalled area was manually 358 identified during a ground-based pre-check. To document this area in a more detailed manner, 10 
359 additional images were taken from a distance of $10 \mathrm{~m}$ away via take-off $\mathrm{C}$. Using the chart in

360 Figure 6, the GSD is less than $5 \mathrm{~mm} /$ pixel. A total of 295 images were acquired during the 3 flights,

361 and all images were used for the 3D reconstruction. As ground-based access (for verification) was

362 not possible from the north side, data collection efforts were concentrated on the bridge's southern

363 side.

364

365

366

367 372 and required approximately 1.5 hours in total. As the bridge deck was not accessible, the terrestrial

373 laser scan data only covered the side of the bridge.

Fig. 17. Flight path on both sides (image showing south side of arches 1-6)

Table 2. Flight Information

\begin{tabular}{lllllll}
\hline Take off & Take-off location & Flight Time & Images & Distance & Height & Angle \\
\hline \multirow{3}{*}{ A } & & & 30 & $20 \mathrm{~m}$ & $20 \mathrm{~m}$ & $0^{\circ}$ \\
& South-east side & \multirow{2}{*}{11 minutes } & 17 & $40 \mathrm{~m}$ & $25 \mathrm{~m}$ & $30^{\circ}$ \\
& & & 24 & $40 \mathrm{~m}$ & $45 \mathrm{~m}$ & $45^{\circ}$ \\
B & & & 33 & $20 \mathrm{~m}$ & $20 \mathrm{~m}$ & $0^{\circ}$ \\
& South-west side & \multirow{2}{*}{14 minutes } & 25 & $40 \mathrm{~m}$ & $25 \mathrm{~m}$ & $30^{\circ}$ \\
C & & & 29 & $40 \mathrm{~m}$ & $45 \mathrm{~m}$ & $45^{\circ}$ \\
& South-east side & 3 minutes & 10 & $10 \mathrm{~m}$ & $20 \mathrm{~m}$ & $0^{\circ}$ \\
\hline
\end{tabular}

\section{TLS Data Collection}

For collection of reference data, a Leica Scan Station P20 terrestrial laser scanner was used (Figure 18). The unit's resolution was set as $12.5 \mathrm{~mm}$ at $10 \mathrm{~m}$ resulting in a typical data density of 6400 $\mathrm{pts} / \mathrm{m}^{2}$. Scans were taken from 3 locations (see Figure 19) along the southeast portion of the bridge

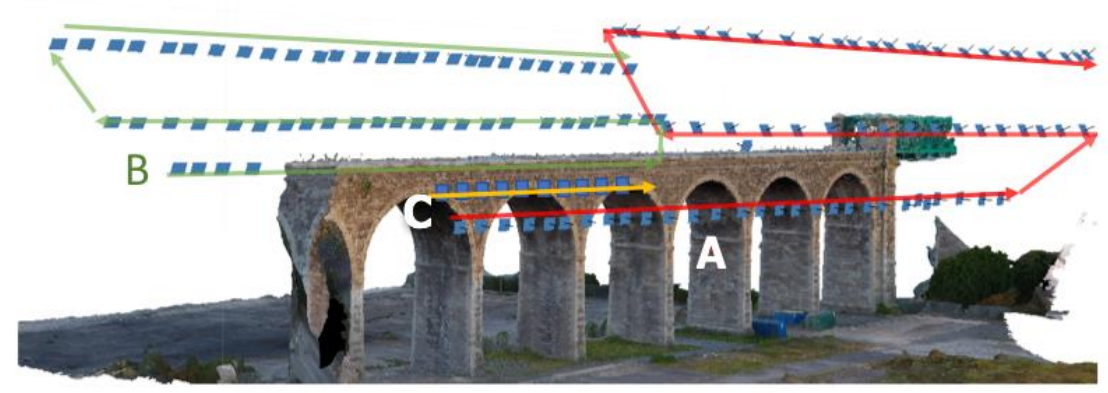




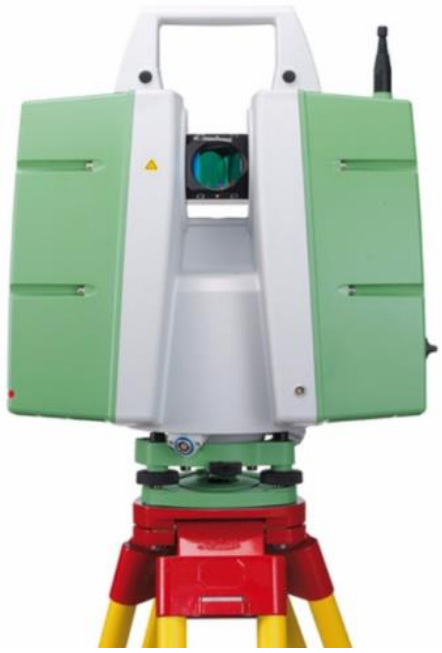

Fig. 18. Terrestrial laser scanner

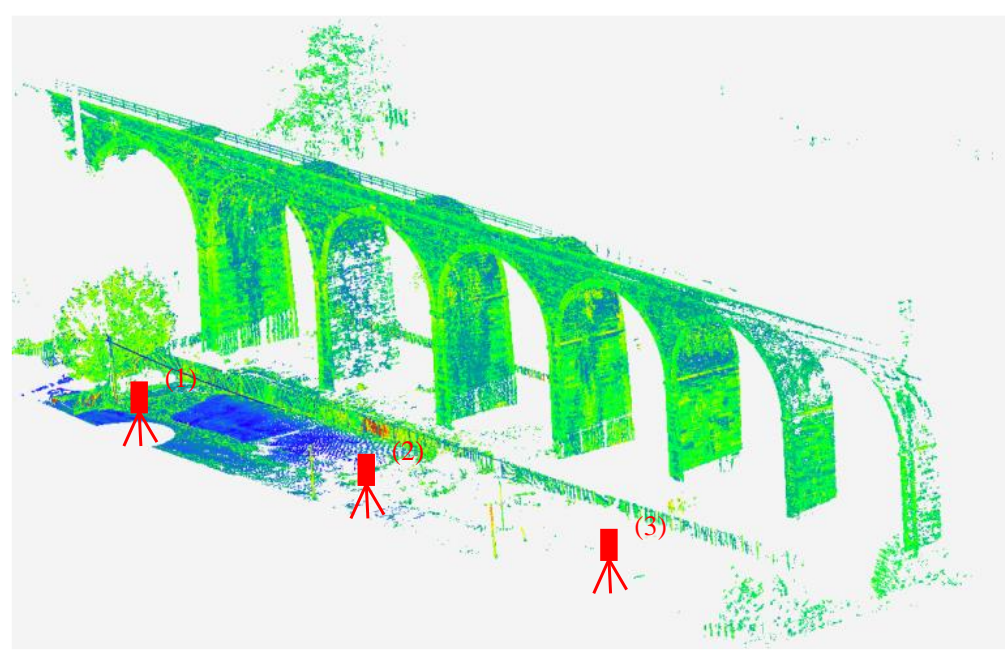

Fig. 19. Scanning location

\section{3D Reconstruction}

376 After data collection, the software Photoscan (Agisoft 2017) was applied to generate the 3D point

377 cloud, including the 153 images from take offs 1 and 2. A Dell laptop with an Intel i7 processor (4 378 cores, $2.8 \mathrm{GHz}$ ), $16 \mathrm{~Gb}$ RAM was employed for the data processing. A total of 4 hours and 14 379 minutes was required to build a model from $24,404,204$ points using UAV-20m (20 m was the 380 closest distance to the object). Adding 10 extra images of arch No. 5 (taken from $10 \mathrm{~m}$ ) increased 381 the dataset to $24,802,421$ points. This resulted in the UAV-10m model (closest distance to object $38210 \mathrm{~m}$ ) which required 5 hours 58 minutes of processing time. As each new image must be matched 383 with all the previous ones in the data set, the additional time is disproportional to the amount of 384 information added (i.e. less than a $2 \%$ increase in the number of points for nearly a $41 \%$ increase 385 in processing time). 


\section{Quality Evaluation}

390 To reduce the computing time of the evaluation, the data related to arch No. 5 (Figure 20) was

391 manually segregated for the additional processing. The three subsets used as input for the

392 evaluation are shown in Figure 21.

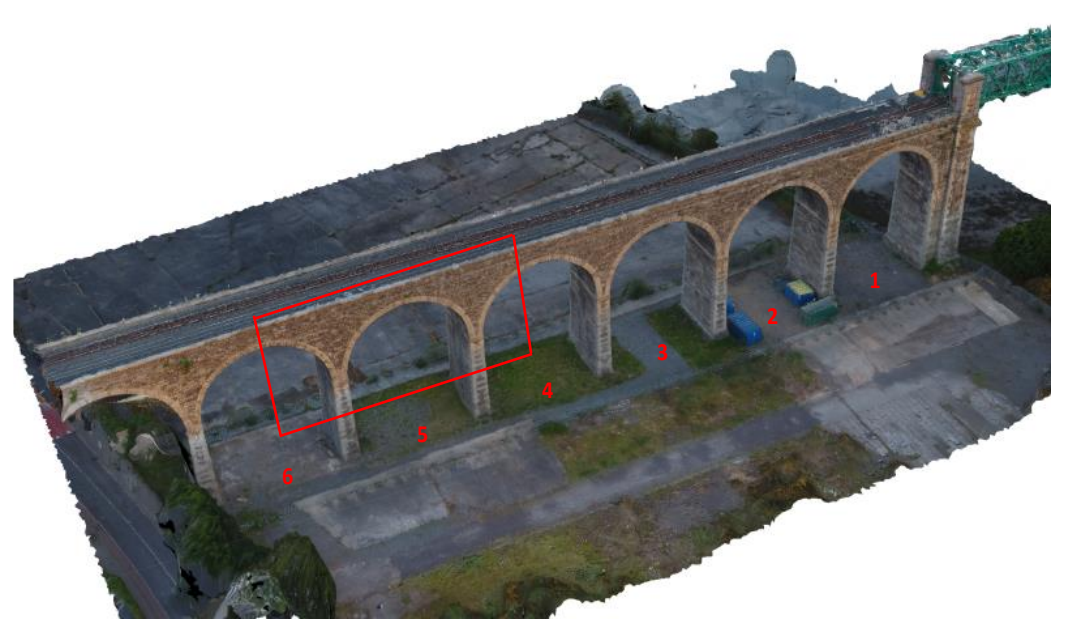

Fig. 20. Model UAV-20m

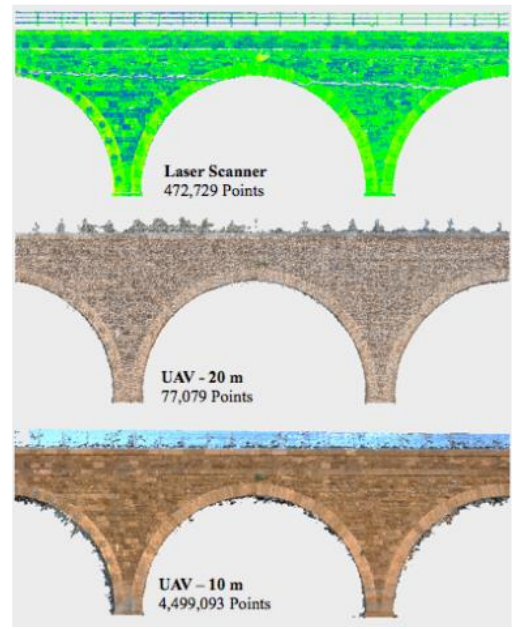

Fig. 21. Evaluation Section for Arch No. 5 (South Side)

\section{Evaluation of Incomplete Data}

395 The TLS dataset was used for defining the valid area of the structure. Calculating the coverage

396 rate involved setting the threshold $\alpha_{\mathrm{p} 2 \mathrm{p}}$ to about 20 times larger than that of $\beta_{\text {ave }}$ to obtain the ground

397 truth and setting it equal to $\beta_{\text {ave }}+\beta_{\text {std }}$ to determine the real coverage. The results are shown in Table

398 3, with the UAV-10m dataset resulting in the best coverage rate at $93.46 \%$. For the UAV-20m

399 dataset, about $20 \%$ of the area was not covered, which largely corresponded to the missing data

400 for the railing portion of the bridge which resulted insufficient feature matching in this area. 
Table 3. Coverage Comparison

\begin{tabular}{cccccc}
\hline Datasets & $\beta$ ave & $\beta$ std & $\alpha \mathrm{p} 2 \mathrm{p}$ & $\begin{array}{c}\text { Area } \\
\mathrm{m}^{2}\end{array}$ & Coverage \\
\hline Ground Truth & 0.0205 & 0.0064 & 0.4101 & 296.7946 & $100 \%$ \\
TLS & 0.0205 & 0.0064 & 0.0269 & 239.6658 & $71.83 \%$ \\
UAV-10m & 0.0078 & 0.0032 & 0.0109 & 277.3921 & $93.46 \%$ \\
UAV-20m & 0.0507 & 0.0174 & 0.0681 & 239.6658 & $80.75 \%$ \\
\hline
\end{tabular}

404

406 To evaluate the point distribution situation with a neighbourhood of a radius $0.05 \mathrm{~m}$, the volume 407 density was calculated for each point (see Figure 22). As expected, TLS point distribution was 408 highly non-uniform, with portions of the bridge closer to the scanner captured more densely (e.g. 409 the bottom left-hand corner) than those further afield. In contrast, the UAV datasets were more 410 uniformly distributed but had more local density variation (as shown in the colour changes in the 411 UAV density maps, especially near the bottom edges or the arches). The density of the $10 \mathrm{~m}$ dataset 412 was higher than the $20 \mathrm{~m}$ dataset, with significant differences between the background and the 413 rails, which can be used as a feature to remove the background noise.

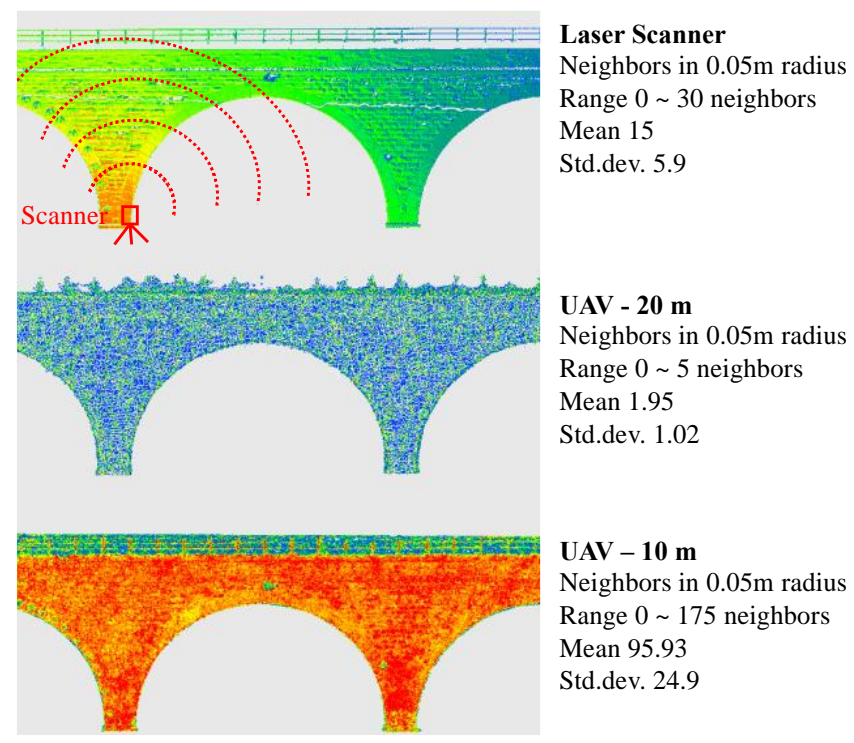

Fig. 22. Point density map 
417 Using the method outlined in the Methodology section, the UAV dataset was aligned with the TLS

418 data, and the outlier noise level for each UAV dataset was calculated (Table 4). The UAV-10m

419 noise level was $4.52 \%$ - approximately 1/3rd less than that of the UAV-20m dataset (at $6.87 \%$ ),

420 which means adding close up images with more details can help reduce the outlier noise level in

421 the reconstructed point cloud.

422

423 Table 4. Outlier Noise Evaluation

\begin{tabular}{ccccccc}
\hline Datasets & $\lambda$ ave & $\lambda$ std & $\alpha \mathrm{c} 2 \mathrm{c}$ & $\begin{array}{c}\text { Total } \\
\text { Points }\end{array}$ & $\begin{array}{c}\text { Outlier } \\
\text { Points }\end{array}$ & $\begin{array}{c}\text { Outlier } \\
\text { Noise } \\
\text { Percentage }\end{array}$ \\
\hline $\begin{array}{c}\text { UAV- } \\
\text { 10m }\end{array}$ & 0.0456 & 0.0705 & 0.1866 & $4,296,232$ & 194,068 & $4.52 \%$ \\
$\begin{array}{c}\text { UAV- } \\
20 \mathrm{~m}\end{array}$ & 0.0784 & 0.1146 & 0.3076 & 73,342 & 5,042 & $6.87 \%$ \\
\hline
\end{tabular}

426 As previously mentioned, measuring surface deviation is easier on a flat surface. In the small,

427 immediate neighbourhood around the checking points, the surface approximates a flat surface.

428 Therefore, using that surface, 20 points were picked randomly for evaluation (Figure 23). The

429 thickness of the UAV-based dataset was about three times greater than that of the TLS data

430 meaning that the TLS data had fewer surface deviations and more closely captured the real surface

431 geometry (Figure 24). Geometric accuracy is especially important for baseline documentation and 432 crack tracking. 


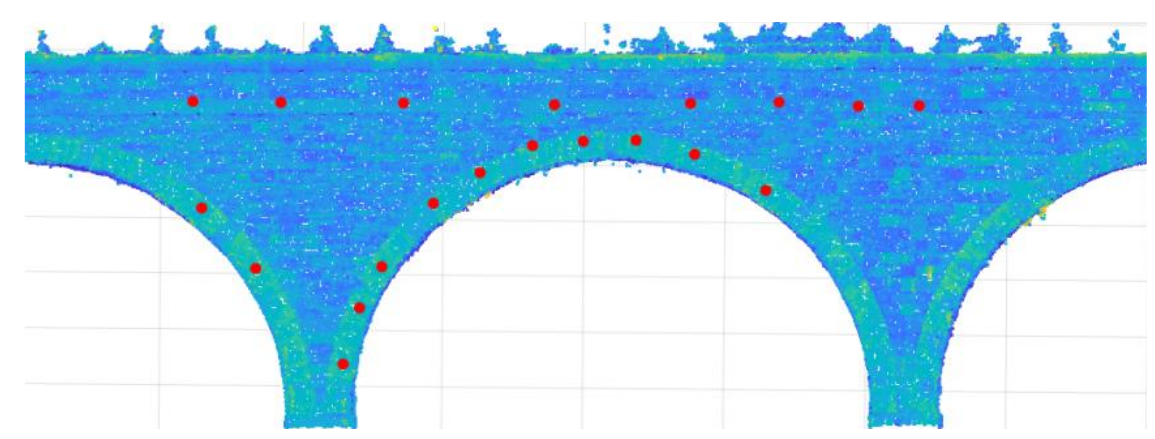

Fig. 23. Random checking points on flat surfaces

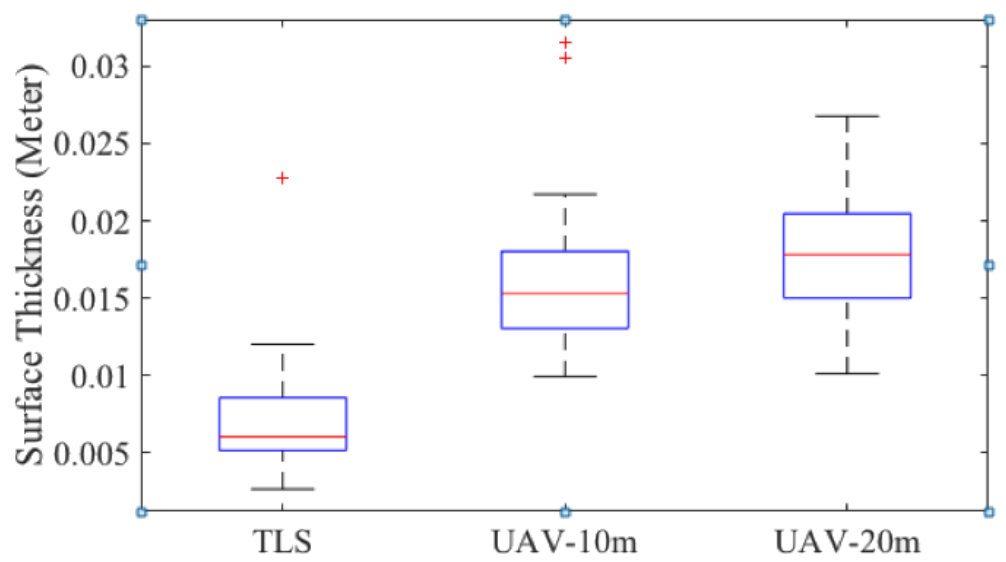

Fig. 24. Thickness distribution

Evaluation of Geometric Accuracy

439 Employing Koutsoudis et al.'s (2014) method for evaluation of geometric accuracy, the TLS data 440 served as a reference data set against which to evaluate the UAV-based point cloud. To measure 441 the point-to-point distance, three easily detectable features were selected. These were corner points 442 at the bottom or top of the arch (Figure 25). As selecting the exact same points across datasets is 443 unlikely, concepts from the "Guide to the Expression of Uncertainty in Measurement" (GUM) 444 were applied (JCGM/WG 1). Each distance was measured 10 times, which was used to calculate 445 the mean distance and the type A standard uncertainty at each location. Table 5 shows the 
446 geometric offset from the UAV-10m dataset with relative errors up to $0.4 \%$, while the UAV-20m 447 dataset had slightly more, with errors up to $0.97 \%$.

448

449

450 Table 5. Point-to-point distance (meter)

\begin{tabular}{l|lll|lll|lll}
\hline \multirow{2}{*}{$\#$} & \multicolumn{3}{|c|}{ UAV-10 } & \multicolumn{3}{c|}{ UAV-20 } & \multicolumn{3}{c}{ TLS } \\
\hline 1 & AB & BC & CA & AB & BC & CA & AB & BC & CA \\
2 & 14.103 & 13.611 & 20.666 & 14.295 & 13.723 & 20.744 & 14.176 & 13.517 & 20.685 \\
3 & 14.080 & 13.590 & 20.668 & 14.343 & 13.776 & 20.799 & 14.175 & 13.567 & 20.672 \\
4 & 14.160 & 13.590 & 20.666 & 14.252 & 13.625 & 20.729 & 14.206 & 13.521 & 20.672 \\
5 & 14.126 & 13.605 & 20.674 & 14.141 & 13.625 & 20.747 & 14.187 & 13.567 & 20.673 \\
6 & 14.120 & 13.626 & 20.657 & 14.295 & 13.723 & 20.747 & 14.197 & 13.566 & 20.681 \\
7 & 14.120 & 13.578 & 20.657 & 14.430 & 13.723 & 20.599 & 14.181 & 13.564 & 20.704 \\
8 & 14.110 & 13.576 & 20.691 & 14.206 & 13.669 & 20.760 & 14.192 & 13.566 & 20.681 \\
9 & 14.156 & 13.563 & 20.636 & 14.345 & 13.625 & 20.801 & 14.179 & 13.561 & 20.692 \\
10 & 14.142 & 13.523 & 20.670 & 14.220 & 13.679 & 20.725 & 14.175 & 13.567 & 20.673 \\
& 14.149 & 13.556 & 20.657 & 14.294 & 13.723 & 20.747 & 14.183 & 13.585 & 20.692 \\
\hline Average & 14.13 & 13.58 & 20.66 & 14.28 & 13.69 & 20.74 & 14.19 & 13.56 & 20.68 \\
Std. Dev & 1.24 & 1.08 & 3.22 & 1.29 & 1.11 & 3.24 & 1.26 & 1.07 & 3.22 \\
Std. Err & 0.39 & 0.34 & 1.02 & 0.41 & 0.35 & 1.02 & 0.40 & 0.34 & 1.02 \\
Distance & $14.1 \pm 0.4$ & $13.6 \pm 0.3$ & $20.7 \pm 1$ & $14.3 \pm 0.4$ & $13.7 \pm 0.4$ & $20.7 \pm 1$ & $14.2 \pm 0.4$ & $13.6 \pm 0.3$ & $20.7 \pm 1$ \\
Relative Err & 0.06 & -0.02 & 0.02 & -0.09 & -0.13 & -0.06 & & - & - \\
Uncertainty & $(0.41 \%)$ & $(0.18 \%)$ & $(0.28 \%)$ & $(0.68 \%)$ & $(0.97 \%)$ & $(0.28 \%)$ & & - & \\
\end{tabular}

Fig. 25. Selected feature points

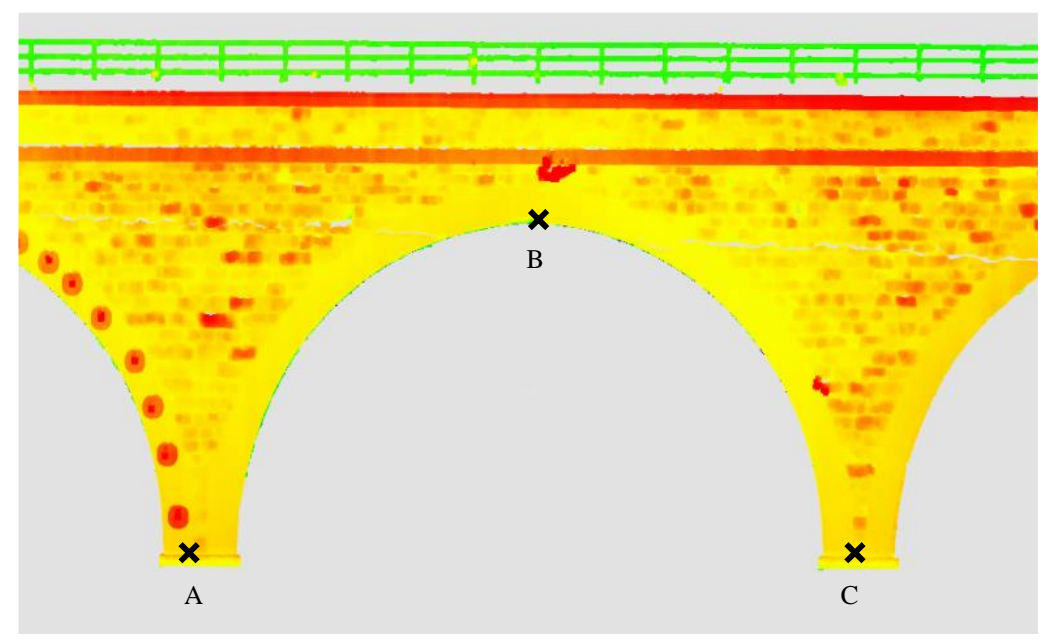




\section{Damage Evaluation}

454 During the pre-check, spalling on arch No. 5 was observed by the inspector visually from the 455 ground (Figure 26a). To measure the volume of the missing area (Figure 26b), the damage 456 evaluation method discussed above was applied. First, the damaged boundary was manually 457 extracted. Then, within the boundary, random points were generated to fill the space (Figure 26c). 458 Finally, a triangular mesh was generated across the damaged part for 3D volume calculation 459 (Figure 26d). The results are shown in Table 6 with only a $3.97 \%$ difference from the UAV-10m 460 dataset, and a $25 \%$ difference from the UAV-20m dataset, thereby showing the critical importance 461 of having high quality data in areas of damage.

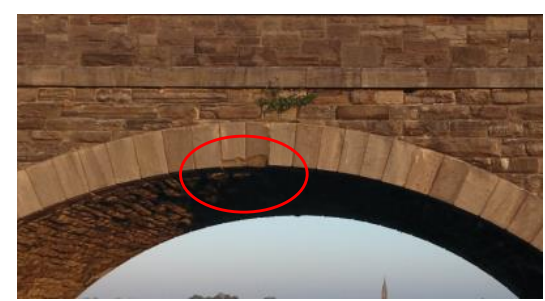

a

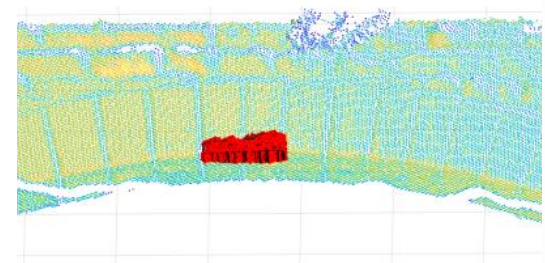

C

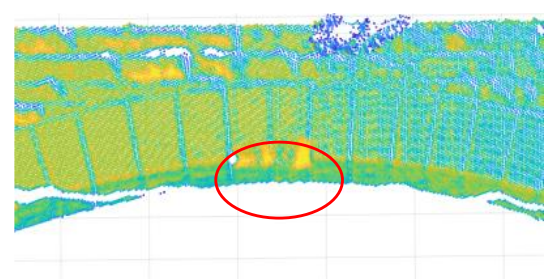

$\mathrm{b}$

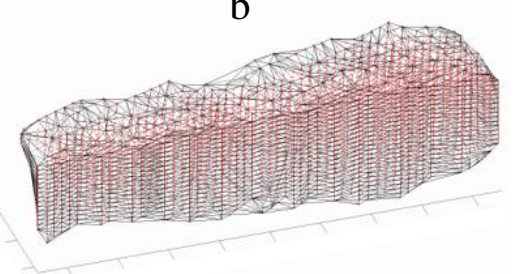

d

Fig. 26. Damage and volume evaluation of spalled brick of south side of arch No. 5. (a) Image

464 data; (b) Point cloud data; (c) Filling of the damaged area with points; (d) Resulting volume of 
Table 6. Volume accuracy evaluation

\begin{tabular}{lll}
\hline & Volume & Error \\
\hline TLS & $0.0151 \mathrm{~m} 3$ & - \\
UAV-10m & $0.0157 \mathrm{~m} 3$ & $3.97 \%$ \\
UAV-20m & $0.0189 \mathrm{~m} 3$ & $25.2 \%$ \\
\hline
\end{tabular}

471

\section{Discussion}

473 As mentioned above, the possibility of using UAVs for bridge inspection has been demonstrated

474 in other studies. While much of the focus of that work centers on using high-end equipment to 475 achieve better results, this is not necessarily so. For example, Katz (2018) reported the output of 476 the relatively low-end DJI phantom series (as was used in the study herein) as comparable to a $477 \$ 70,000$ Trimble unit with land-surveyor accuracy levels. Nonetheless, point clouds generated by 478 the UAV-SfM method are generally less accurate than the TLS data. For example, while Slocum 479 and Parrish (2017) showed that, under idealized conditions, UAV-SfM inspection accuracy can be 480 in the range of $2.6 \mathrm{~mm}$ to $32.2 \mathrm{~mm}$, field experiments have shown that $3 \mathrm{D}$ distance measurement 481 errors are more typically at the sub-meter level (Mosbrucker et al. 2017). Similarly, under idealized 482 conditions, sales brochures claim that TLS can achieve mm level accuracy, but field experiments 483 ultimately demonstrate accuracy at the centimeter level (Quagliarini et al. 2017), which is a slight, 484 but notable improvement upon the sub-meter accuracy of UAV-SfM in the field. However, accuracy is only one aspect of a quality dataset appropriate for inspection. There are 487 also considerations of direct costs, scheduling issues, and access. For example, in the case study 488 presented herein, the UAV equipment costs were less than $3 \%$ of that of the TLS (\$2500 vs $489 \$ 103,000$ ), and the on-site survey time was 33\% (1 hour for UAV and 3 hours for TLS (Table 7). 
Table 7. Comparison of UAV to TLS inspection

\begin{tabular}{l|l|l}
\hline & UAV & TLS (Leica P-20) \\
\hline Equipment and software costs & $\$ 2,500$ & $\$ 103,000^{*}$ \\
\hline Data Collection Time & $<1 \mathrm{~h}$ & $3 \mathrm{~h}$ \\
\hline Data Processing Time & $4 \mathrm{~h}-1$ day & $1 \mathrm{~h}$ \\
\hline Data Completeness & $>80 \%$ & $71.83 \%$ \\
\hline Point Distribution & Well distributed & Radially distributed \\
\hline Outlier Noise level & High & Low \\
\hline Surface Deviation & High & Low \\
\hline Geometry Accuracy & Centimetre Level & Millimetre level \\
\hline
\end{tabular}

*Relatively high quality units can be obtained for as little as $\$ 25,000$

494 While these factors are important, for bridge projects access issues can predominate. Although the 495 TLS data are more accurate, the scanner could only be set on bank. As mentioned before, the TLS 496 data will cause a radial distribution problem in this situation. The data quality for the mid-span of 497 the bridge will be relative poor, as it is far from the scanner and negatively impacted by the angle 498 of incidence caused by the scanner position (Laefer et al. 2009). Positioning can also cause over499 estimation of crack widths and lengths (Laefer et al. 2010) and has some strong practical limits 500 based on positioning and beam size, even from only $15 \mathrm{~m}$ (Laefer et al. 2014). Additionally, line501 of-site obstacles and uneven surfaces will interfere with complete coverage in the TLS dataset. 502 The offset distance and angle of incidence has also been shown to compromise the data damage 503 collection process. In contrast UAV-based 3D reconstruction method can easily overcome those 504 problems and generate a full covered uniform point cloud with thoughtful pre-flight path planning.

506 Unfortunately, there are also disadvantages to UAV-based inspection. In the case herein, the UAV-

507 based point cloud had a higher noise level than the TLS-based one, which was reflected in a more 508 than 3 times higher deviation in the structure surface and marginally more outlier points (more 509 than 4.52\%). Additionally, narrow features make key point matching difficult using an SfM 
510 method, which will cause problems for bridge cable or truss inspection. Moreover, the 3D

511 reconstruction process is more time-consuming than the TLS post-processing - spanning from a

512 few hours to several days for the point cloud generation for each of the 3 flights versus only a

513 single hour for the TLS data.

\section{Conclusions}

516 With respect to bridge inspection, this paper introduced a blended UAV-SfM method for imagery 517 acquisition and 3D reconstruction. A case study for a major bridge in Dublin, Ireland was 518 presented, and the proposed UAV-SfM method was compared with TLS-based inspection. A series

519 of data evaluation methods were proposed to evaluate the point cloud performance in data 520 completeness, density distribution, outlier noise level, surface deviation and geometry accuracy.

521 In general, the study demonstrated that the UAV-SfM method can offer significant advantages in 522 equipment cost, surveying time, point distribution, and ultimate data coverage. However, problems 523 remain including high noise levels, low geometry accuracy and long post-processing times.

525 To solve these problems, future research will need to focus on optimizing 3D reconstruction 526 algorithms and developing better noise removal techniques. Possible solutions could involve 527 feature extraction algorithms that incorporate UAV position and orientation based on internal 528 Global Position System (GPS) data and inertial measurement units, which could involve applying 529 a weighting function to emphasize target features and de-emphasize items likely to be in the 530 background (e.g. ground and sky) based on the proximity and focal area. Noise may similarly be 531 removed through objective-based clustering algorithms. 


\section{Acknowledgments}

534 This project was made possible through the generous support of the European Union's Horizon 5352020 Research and Innovation programme under the Marie Skłodowska-Curie grant 642453.

\section{References}

538 AASHTO (American Association of State Highway and Transportation Officials). (1970). Manual for Maintenance Inspection of Bridges, vol. 19705. AASHTO: Washington, DC,

Agisoft. (2017). “Agisoft PhotoScan.” <http://www.agisoft.com/> (Feb. 20, 2018)

Berger, M., Alliez, P., Tagliasacchi, A., Seversky, L. M., Silva, C. T., Levine, J. A. and Sharf, A. (2014). "State of the Art in Surface Reconstruction from Point Clouds." Eurographics 2014 -- State of the Art Reports, 1(1), 161-185, 10.2312/egst.20141040

Besl, P.J., and McKay, N. D. (1992). "A Method for Registration of 3-D Shapes.” IEEE Transactions on Pattern Analysis and Machine Intelligence, 14(239-56), 
Byrne, J., Laefer, D. F. and O’Keeffe, E. (2017a). “Maximizing Feature Detection in Aerial Unmanned Aerial Vehicle Datasets.” Journal of Applied Remote Sensing, 11(2), 1-2, 10.1117/1.JRS.11.025015.

Byrne, J., O'Keeffe, E., Lennon, D., and Laefer, D. F. (2017b). “3D Reconstructions Using Unstabilized Video Footage from an Unmanned Aerial Vehicle.” Journal of Imaging, 3(2), 15, 10.3390/jimaging3020015.

Chan, Br., Guan, H., Jo, J., and Blumenstein, M. (2015). “Towards UAV-Based Bridge Inspection Systems: A Review and an Application Perspective.” Structural Monitoring and Maintenance, 2(3), 283-300, 10.12989/smm.2015.2.3.283.

Chen, S., Laefer, D. F., Byrne, J., and Natanzi, A. S. (2017). "The Effect of Angles and Distance on Image-Based, Three-Dimensional Re-Constructions." Proc. European Safety and Reliability ESREL2017, Taylor \& Francis, Abingdon, UK, 2757-61.

Chen, S., Laefer, D. F., and Mangina, E. (2016). "State of Technology Review of Civilian UAVs." Recent Patents on Engineering, 10(3), 160-74, $10.2174 / 1872212110666160712230039$.

Cheng, S.-W., and Lau, M.-K. (2017). "Denoising a Point Cloud for Surface Reconstruction.” arXiv Preprint, arxiv:1704.04038v1.

Díaz-Vilariño, L., González-Jorge, H., Martínez-Sánchez, J., Bueno, M., and Arias, P. (2016). "Determining the Limits of Unmanned Aerial Photogrammetry for the Evaluation of Road Runoff.” Measurement, 85, 132-41, 10.1016/j.measurement.2016.02.030.

Eschmann, C., Kuo, C.M., Kuo, C.H., and Boller, C. (2013). "High-Resolution Multisensor Infrastructure Inspection With Unmanned Aircraft Systems." International Archives of 
the Photogrammetry, Remote Sensing and Spatial Information Sciences, XL-1/W2, 12529, 10.5194/isprsarchives-XL-1-W2-125-2013.

Escobar-wolf, R., Oommen, T., Brooks, C. N., Dobson, R. J., and Ahlborn, T. M. (2017). “Unmanned Aerial Vehicle (UAV)-Based Assessment of Concrete Bridge Deck Delamination Using Thermal and Visible Camera Sensors: A Preliminary Analysis.” Research in Nondestructive Evaluation, 1-16, 10.1080/09349847.2017.1304597.

Fernandez Galarreta, J., Kerle, N., and Gerke, M. (2014). “UAV-Based Urban Structural Damage Assessment Using Object-Based Image Analysis and Semantic Reasoning.” Natural Hazards and Earth System Sciences Discussions, 2(9), 5603-45,

Hallermann, N., and Morgenthal, G. (2016). "From Aerial Photography to 3-Dimensional Inspection of Bridges." Proc. IABSE Conference Guangzhou 2016: Bridges and Structures Sustainability-Seeking Intelligent Solutions, International Association for Bridge and Structural Engineering, Zurich, 546-53, 10.2749/222137816819258898.

Hassanalian, M, and Abdelke, A. (2017). "Classifications, Applications, and Design Challenges of Drones: A Review.” Progress in Aerospace Sciences, 91(May), 99-131, 10.1016/j.paerosci.2017.04.003.

Hinks, T., Carr, H., and Laefer, D. F. (2009). “Flight Optimization Algorithms for Aerial LiDAR Capture for Urban Infrastructure Model Generation.” Journal of Computing in Civil Engineering, 10.1061/(ASCE)0887-3801(2009)23:6(330).

Huang, H., Li, D., Zhang, H., Ascher, U., and Cohen-Or, D. (2009). "Consolidation of Unorganized Point Clouds for Surface Reconstruction.” ACM Transactions on Graphics (TOG), 28(5), 176, 10.1145/1618452.1618522. 
Jahanshahi, M. R., Masri, S. F., and Sukhatme, G. S. (2011). "Multi-Image Stitching and Scene Reconstruction for Evaluating Defect Evolution in Structures." Structural Health Monitoring, 10(213), 643-57, 10.1177/1475921710395809.

JCGM/WG 1 (Working Group 1 of the Joint Committee for Guides in Metrology). (2008). "Evaluation of Measurement Data — Guide to the Expression of Uncertainty in Measurement." JCGM 100:2008, Online. <https://www.bipm.org/utils/common/documents/jcgm/JCGM_100_2008_E.pdf>

Katz, Daniel. (2018). "Distinctive Image Features from Scale-Invariant Keypoints." Commercial UAV News, May 16. <https://www.expouav.com/news/latest/surveyors-and-otherprofessional-drone-operators-should-be-focused-on-accuracy-and-reliability/>.

Kim, J.-W., Kim, S.-B., Park, J.-C. and Nam, J. -W. (2015). “Development of Crack Detection System with Unmanned Aerial Vehicles and Digital Image Processing.” Proc. Advances in Structure Engineering and Mechanics, Incheon, Korea, 1-11.

Koutsoudis, A., Vidmar, B., Ioannakis, G., Arnaoutoglou, F., Pavlidis, G., and Chamzas, C. (2014). “Multi-Image 3D Reconstruction Data Evaluation.” Journal of Cultural Heritage, 15(1), 73-79, 10.1016/j.culher.2012.12.003.

Laefer, D.F., Fitzgerald, M., Maloney, E.M., Coyne, D., Lennon, D., and Morrish, S. (2009). "Lateral Image Degradation in Terrestrial Laser Scanning Author(s)." Structural Engineering International, 19(2), 184-89, 10.2749/101686609788220196.

Laefer, D.F., Gannon, J. and Deely, E. (2010). "Reliability of crack detection for baseline condition." Journal of Infrastructure Systems, 16(2), 129-137.

Laefer, D.F., Truong-Hong, L., Carr, H. and Singh, M. (2014). "Crack detection limits in unit based masonry with terrestrial laser scanning." NDT\&E International, 62, 66-76. 
623 Lowe, D.G. (2004). “Distinctive Image Features from Scale Invariant Keypoints.” Int'l Journal of Computer Vision, 60(2), 91-110, 10.1023/B:VISI.0000029664.99615.94.

625 Lueker, M., and Marr, J. (2014). “Scour Monitoring Technology Implementation.” MN/RC 201437, Minnesota Department of Transportation, St. Paul, MN.

627 Moenning, C., and Dodgson, N.A. (2003). "A New Point Cloud Simplification Algorithm.” Proc. 3rd IASTED International Conference on Visualization, Imaging, and Image

Mosbrucker, A.R., Major, J.J., Spicer, K.R., and Pitlick, J. (2017). “Camera System Considerations for Geomorphic Applications of SfM Photogrammetry." Earth Surface Processes and Landforms, 42(6), 969-86, 10.1002/esp.4066.

Muja, M., and Lowe, D.G. (2009). "Fast Approximate Nearest Neighbors with Automatic

Nishimura, S., Kimoto, K., Abe, A., and Okazawa, T. (2012). "Development of a Hybrid Camera System for Bridge Inspection.” Proc. Sixth International IABMAS Conference, Taylor \& Francis, Abingdon, UK, 2197-2203. Algorithm Configuration.” Proc. VISAPP International Conference on Computer Vision Theory and Applications, Scitepress, Setubal, Portugal, 331-40.

644

Paine, D.P., and Kiser, J.D. (2003). Aerial photography and image interpretation. John Wiley \& Sons, Hoboken, NJ.

Palmer, L. M., Franke, K. W., Martin, R. A., Sines, B. E., Rollins, K. M. and Hedengren, J. D. (2015). "The Application and Accuracy of Structure from Motion Computer Vision Models with Full- Scale Geotechnical Field Tests.” IFCEE 2015, 2432-41, $10.1061 / 9780784479087.225$. 
645 Quagliarini, E., Clini, P., and Ripanti, M. (2017). "Fast, Low Cost and Safe Methodology for the 646 Assessment of the State of Conservation of Historical Buildings from 3D Laser Scanning : The Case Study of Santa Maria in Portonovo (Italy).” Journal of Cultural Heritage, 24, 175-83, 10.1016/j.culher.2016.10.006.

RAIU (Railway Accident Investigation Unit). (2010). "Malahide Viaduct Collapse on the Dublin to Belfast Line, on the 21 St August 2009." Investigation Report No. 2010 - R004, RAIU, Blackrock, Ireland.

Sargent, I., Harding, J., and Freeman, M. (2007). "Data Quality in 3D: Gauging Quality Measures from Users’ Requirements.” International Archives of Photogrammetry, Remote Sensing and Spatial Information Sciences, 36(2/C43), 8.

Schonberger, J.L., and Frahm, J.-M. (2016). “Structure-from-Motion Revisited.” In Proceedings of the IEEE Conference on Computer Vision and Pattern Recognition, 4104-13.

Slocum, R.K., and Parrish, C.E. (2017). "Simulated Imagery RenderingWorkflow for UASBased Photogrammetric 3D Reconstruction Accuracy Assessments.” Remote Sensing, 9(936), 1-19, 10.3390/rs9040396.

Sterritt, G. (2009). "Review of Bridge Inspection Competence and Training Project Report." Project: UG637, UK Bridges Board, London, UK.

Szeliski, R. (2010). Computer Vision: Algorithms and Applications. Springer-Verlag, London, UK, 10.1007/978-1-84882-935-0.

Tagliasacchi, A., Zhang, H., and Cohen, D. (2009). "Curve Skeleton Extraction from Incomplete Point Cloud.” ACM Transactions on Graphics, 27(3), 1-10, 10.1145/1531326.1531377. 
666

667

668

669

670

671

672

673

674

675

676

677

678

679

680

681

682

683

684

685

686

Truong-Hong, L., Falter, H., Lennon, D., and Laefer, D. F. (2016). "Framework for Bridge Inspection with Laser Scanning.” Proc. EASEC-14 Structural Engineering and Construction, Ho Chi Minh City, Vietnam, 6 - 8 January 2016, 1-9.

Truong-Hong, L., and Laefer, D. F. (2015). “Documentation of Bridges by Terrestrial Laser Scanner.” In IABSE Symposium Report (Vol. 105, No. 19), International Association for Bridge and Structural Engineering, 1-8.

Ullman, S. (1979). “The Interpretation of Structure from Motion.” Proceedings of the Royal Society of London, Series B, Biological Sciences, 203(1153), 405-26.

Wallace, L., Lucieer, A., Turner, D., and Vop, P. (2016). “Assessment of Forest Structure Using Two UAV Techniques: A Comparison of Airborne Laser Scanning and Structure from Motion (SfM) Point Clouds.” Forests, 7(62), 1-16, 10.3390/f7030062.

Wallace, L., Lucieer, A., Watson, C., and Turner, D. (2012). "Development of a UAV-LiDAR System with Application to Forest Inventory.” Remote Sensing, 4(6), 1519-43, $10.3390 /$ rs4061519.

Westoby, M. J., Brasington, J., Glasser, N. F., Hambrey, M. J., and Reynolds, J. M. (2012). “'Structure-from-Motion' Photogrammetry: A Low-Cost, Effective Tool for Geoscience Applications.” Geomorphology, 179, 300-314, 10.1016/j.geomorph.2012.08.021.

Wolff, K., Kim, C., Zimmer, H., Schroers, C., Botsch, M. and Sorkine-hornung Alexander, O. (2016). "Point Cloud Noise and Outlier Removal for Image-Based 3D Reconstruction." Proc., 2016 Fourth International Conference on 3D Vision Point, IEEE, Piscataway, 118-27, 10.1109/3DV.2016.20. 
687 Yin, Z., Seto, C. and Mao, Y. (2015). "Develop a UAV Platform for Automated Bridge

688 Inspection." Report No. 25-1121-0003-295, Missouri University of Science and

689 Technology, Rolla, MO.

690 Zarco-Tejada, P. J., Diaz-Varela, R., Angileri, V., and Loudjani, P. (2014). “Tree Height

691 Quantification Using Very High Resolution Imagery Acquired from an Unmanned Aerial

692 Vehicle (UAV) and Automatic 3D Photo-Reconstruction Methods.” European Journal of

693 Agronomy, 55, 89-99, 10.1016/j.eja.2014.01.004.

694 